\title{
Implante de válvula aórtica transcatéter transapical: un procedimiento novedoso en el norte peruano
}

\section{Transapical transcatheter aortic valve replacement: a new procedure in Northern Peru}

Correspondencia Carlos A. Zamora-Rodríguez carzamrod@yahoo.com

Recibido: 01/10/2017

Aprobado: 24/01/2018

Citar como: Zamora-Rodríguez CA, León-Burgos AL, Yucra-Lazo J, Ramirez-Zapata JC. Implante de válvula aórtica transcatéter transapical: un procedimiento novedoso en el norte peruano. Acta Med Peru. 2018;35(1):60-4

\author{
Carlos A. Zamora-Rodríguez¹,3 , A. Luis León-Burgos¹, Jorge Yucra-Lazo², Juan \\ Carlos Ramírez-Zapata ${ }^{1}$ \\ ${ }^{1}$ Unidad de Cuidado Crítico Cardiovascular, Hospital de Alta Complejidad Virgen de la Puerta. Trujillo, Perú. \\ ${ }^{2}$ Departamento de Especialidades Médicas, Hospital de Alta Complejidad Virgen de la Puerta. Trujillo, Perú. \\ ${ }^{3}$ Escuela Profesional de Medicina Humana, Universidad Privada Antenor Orrego. Trujillo, Perú
}

\section{RESUMEN}

La estenosis aórtica severa sintomática secundaria a calcificación degenerativa constituye un reto terapéutico si el paciente no es tributario de tratamiento quirúrgico de reemplazo valvular. La colocación de un implante valvular aórtico transcatéter (TAVI) es una alternativa terapéutica para estos casos. Se presenta el caso de un varón de 78 años con antecedentes de hipertensión arterial, enfermedad renal crónica (estadio IIla), tabaquismo pesado, portador de marcapaso definitivo, enfermedad arterial periférica y policitemia vera. A la evaluación, el paciente cursaba con una disnea de clase III (escala NYHA) desde hace un año. El ecocardiograma transtorácico mostró calcificación severa de velos aórticos, una fracción de eyección ventricular izquierda de $44,7 \%$ y un área valvular de $0,58 \mathrm{~cm}^{2}\left(0,31 \mathrm{~cm}^{2} / \mathrm{m}^{2}\right)$; la angiografía mostró enfermedad arterial coronaria moderada y la angiotomografía una calcificación severa de la aorta torácica ("aorta en porcelana"). Por considerarlo de alto riesgo, se realizó colocación de TAVI por vía transapical (válvula bioprotésica Braile Biomédica $N^{\circ} 30$ ), con controles ecocardiográficos satisfactorios. El caso que presentamos constituye el primero realizado en el norte del país.

Palabras clave: Estenosis de la válvula aórtica; Reemplazo de la válvula aórtica transcatéter; Implantación de prótesis de válvulas cardíacas (fuente: DeCS BIREME). 


\begin{abstract}
Severe symptomatic aortic stenosis secondary to degenerative calcification may be a real therapeutic challenge if the patient does not undergo an aortic valve replacement. Transapical transcatheter aortic valve replacement is a valid therapeutic option for these cases. We present the case of a 78-year old male with the following past and current medical history: high blood pressure, chronic kidney disease (stage IIla), heavy tobacco smoking, use of a permanent pacemaker, peripheral arterial disease, and polycythemia vera. When assessed, the patient had had class III heart failure (NYHA classification) for one year. Transthoracic ultrasonography showed severe calcification of the aortic cusps, a $44.7 \%$ left ventricular ejection fraction, and a $0.58 \mathrm{~cm}^{2}\left(0.31 \mathrm{~cm}^{2} / \mathrm{m}^{2}\right)$ valve surface area. Angiography showed moderate coronary heart disease, and angiotomography showed severe calcification of the aortic valve ('porcelain aorta'). Since this patient was considered at high-risk, a transapical transcatheter aortic valve replacement was performed (Braille Biomedical $\mathrm{N}^{\circ}$ 30 bioprosthetic valve), and the ultrasonography controls showed satisfactory results. This case is the first procedure of its kind performed in Northern Peru.

Keywords: Aortic valve stenosis; Transcatheter aortic valve replacement; Heart valve prosthesis implantation (source: MeSH NLM).
\end{abstract}

\section{INTRODUCCIÓN}

La estenosis aórtica es el engrosamiento y la rigidez de la válvula que provoca una disminución del área valvular y es una de las enfermedades degenerativas o asociadas a la edad más frecuente en la población adulta ${ }^{[1,2]}$; alcanza una prevalencia de hasta $4,6 \%$ en pacientes mayores de 75 años ${ }^{[3]}$. Cuando esta valvulopatía se torna sintomática, la tasa de sobrevida a los dos años disminuye al $50 \%$ y al $20 \%$ a los cinco años ${ }^{[4]}$. La cirugía de reemplazo valvular aórtico ha sido el único tratamiento efectivo que permite un aumento en el tiempo de sobrevida y el alivio de los síntomas ${ }^{[5]}$.

La colocación de un implante valvular aórtico transcatéter (TAVI, por sus siglas en inglés) es una novedosa alternativa terapéutica destinada para pacientes sintomáticos con estenosis aortica severa por calcificación degenerativa (estadio D), con alto riesgo de muerte o que no pueden afrontar un cambio valvular quirúrgico; no obstante, se ha ido ampliando a pacientes con riesgo moderado ${ }^{[6]}$.

La colocación del TAVI se realiza con un abordaje retrógrado o anterógrado, dependiendo de la anatomía vascular y algunas condiciones específicas del paciente. En el abordaje retrógrado se utilizan con más frecuencia las vías transfemoral, transsubclavio y transaórtico, mientras que para el anterógrado la vía transapical ${ }^{[7,8]}$. El dispositivo valvular es comprimido y situado en el extremo distal del catéter balón e insertado a través de la arteria femoral u otro vaso o por una pequeña incisión entre las costillas, para avanzar hacia los vasos sanguíneos o el ápice del corazón, respectivamente. Una vez alcanzada la válvula nativa disfuncional, el dispositivo es liberado y anclado, siendo su funcionamiento de cierre y apertura dirigido por el flujo sanguíneo ${ }^{[7]}$.

Los estudios de seguridad y eficacia que avalan el uso de los TAVI iniciaron con los estudios PARTNER I (año 2010) y II (2016). El estudio PARTNER I (cohorte B) se realizó en pacientes con estenosis aórtica severa, no tributarios de cirugía de reemplazo valvular y comparó el TAVI vía transfemoral con el tratamiento estándar, que incluía la valvuloplastía con balón; al año de seguimiento, se encontró que la mortalidad por cualquier causa fue un $20 \%$ menor en pacientes del grupo con TAVI en comparación con los de terapia estándar ${ }^{[9]}$. El estudio PARTNER II comparó pacientes con estenosis aórtica severa con riesgo intermedio, encontrando resultados de no inferioridad en muerte o accidente vascular cerebral isquémico incapacitante a los dos años de seguimiento entre el grupo con TAVI y el grupo que fue sometido a reemplazo valvular quirúrgico ${ }^{[10]}$.

La primera intervención de colocación del TAVI fue realizado en Francia y fue reportado por Cribier en el año $2002^{[11]}$. En el Perú, la colocación del TAVI por vía femoral se inició en el año 2011, y desde el 2015 se ha usado la vía transapical en la ciudad de Lima en forma casi exclusiva. En el año 2017, esta última vía ha sido abordada exitosamente en un establecimiento del norte del Perú y es el caso describimos a continuación.

\section{REPORTE DE CASO}

Presentamos el caso de un varón de 78 años, de etnia asiática, procedente de Chocope (106 $\mathrm{m}$ de altitud), distrito de Ascope, departamento de La Libertad (ubicado en la costa norte del Perú), con antecedentes de hipertensión arterial de larga data, enfermedad renal crónica estadio 3a, tabaquismo pesado, portador de marcapaso definitivo, enfermedad arterial periférica, policitemia vera y alérgico a la penicilina, metamizol y al clopidogrel, quien cursó hace aproximadamente un año con disnea clase funcional III (escala NYHA).

Al examen físico, se auscultaron ruidos cardiacos con S2 disminuido asociado a un soplo mesosistólico en foco aórtico III/VI, con fenómeno de Gallavardin.

El ecocardiograma transtorácico demostró una fracción de eyección del ventrículo izquierdo (FEVI) de 44,7\%, disfunción 

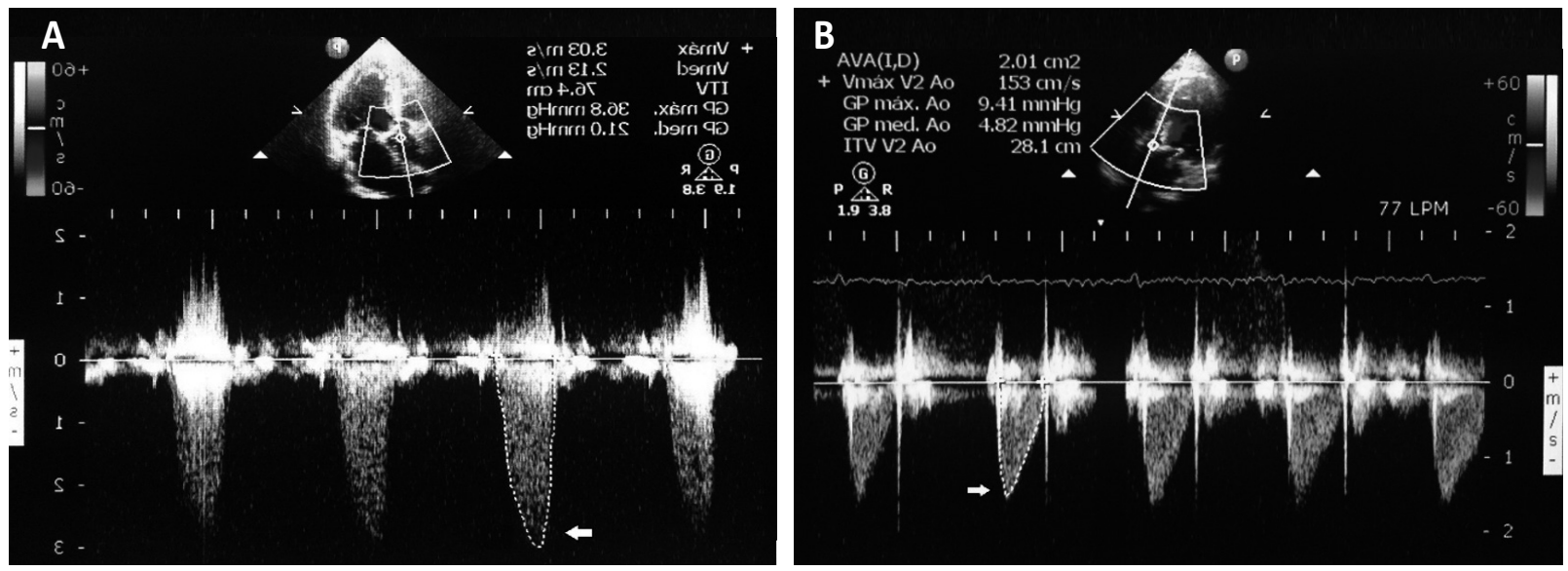

Figura 1. A) Ecocardiograma transtoracico pre-TAVI: en ventana acústica A5C con doppler continuo a nivel de válvula aórtica, se aprecia velocidad máxima y gradiente media. B) Ecocardiograma transtoracico post-TAVI: en ventana acústica A5C con doppler continuo a nivel de válvula implantada bio-protésica en posición aórtica, se aprecia velocidad máxima y gradiente media.

diastólica tipo II, hipertrofia moderada concéntrica del ventrículo izquierdo, en válvula aórtica se encontró una calcificación severa de velos aórticos, con velocidad máxima de $3,03 \mathrm{~m} / \mathrm{s}$, gradiente media de $21 \mathrm{mmHg}$; la área de válvula aórtica (AVA) por ecuación de continuidad estimada fue de $0,58 \mathrm{~cm}^{2}-0,31 \mathrm{~cm}^{2} / \mathrm{m}^{2}$ (Figura $1 \mathrm{~A})$. Se concluyó con el diagnóstico de estenosis aórtica severa con bajo flujo, bajo gradiente.

En la analítica sanguínea se evidenció un valor de hemoglobina de $20 \mathrm{~g} / \mathrm{dL}$, hematocrito de $61 \%$ y un recuento plaquetario de $451 \mathrm{x}$ $10^{9} /$ L. Por sugerencia de Hematología se indicó tratamiento oral con hidroxicarbamida y realización de sangrías por flebotomía de aproximadamente $500 \mathrm{~mL} / \mathrm{vez}$ por tres oportunidades. Luego del tratamiento, se redujo la hemoglobina a $16 \mathrm{~g} / \mathrm{dL}$, hematocrito de $50 \%$ y un recuento plaquetario de $353 \times 10^{9} / \mathrm{L}$. No hubo mejoría sintomatológica.

Se realizó una angiografía coronaria en donde se encontró enfermedad moderada no tributario de revascularización; además, se identificó una calcificación severa de la aorta ascendente.

A la evaluación clínico-quirúrgica se encontró un riesgo previsto de mortalidad de $2,64 \%$ según STS (Society of Thoracic Surgeons), pero no tributario de tratamiento quirúrgico debido a la calcificación severa de la aorta torácica, por lo que se optó por colocación de TAVI por vía transapical.

Previo a la intervención, se realizó un angiotomografía de la aorta torácica para las mediciones requeridas según protocolo (Figura 2): diámetro de la aorta ascendente ( $31 \mathrm{~mm})$, diámetro de la unión sino-tubular (29 mm), anillo aórtico $(28 \times 29 \mathrm{~mm})$, distancia ostium-arteria coronaria derecha $(26,4 \mathrm{~mm})$ y distancia ostium-arteria coronaria izquierda (16 mm). La prueba confirmó una calcificación severa de la aorta torácica en toda su extensión ("aorta en porcelana") (Figuras 3A y 3B).
Para la colocación del TAVI se usó anestesia general inhalatoria, se realizó una mini toracotomía anterolateral izquierda a nivel del sexto espacio intercostal y se implantó una válvula bioprotésica Braile Biomédica $\mathrm{N}^{\circ} 30$ en forma anterógrada bajo visión angiográfica. En aortografía de control, se apreció expansión adecuada de la prótesis (Figura 4A y 4B) con fuga paravalvular leve, que se corroboró por ecocardiograma transesofágico in situ.

Al segundo día se le retiró el dren pericárdico y al tercer día inició deambulación. A la semana del procedimiento se realizó un ecocardiograma transtorácico control encontrando una mejoría de la FEVI del $52 \%$ y a nivel de la válvula bio-protésica una velocidad máxima de $1,53 \mathrm{~m} / \mathrm{s}$, gradiente media de $4,8 \mathrm{mmHg}$ y AVA estimada en $2,0 \mathrm{~cm}^{2}-1,1 \mathrm{~cm}^{2} / \mathrm{m}^{2}$ (Figura 1B).

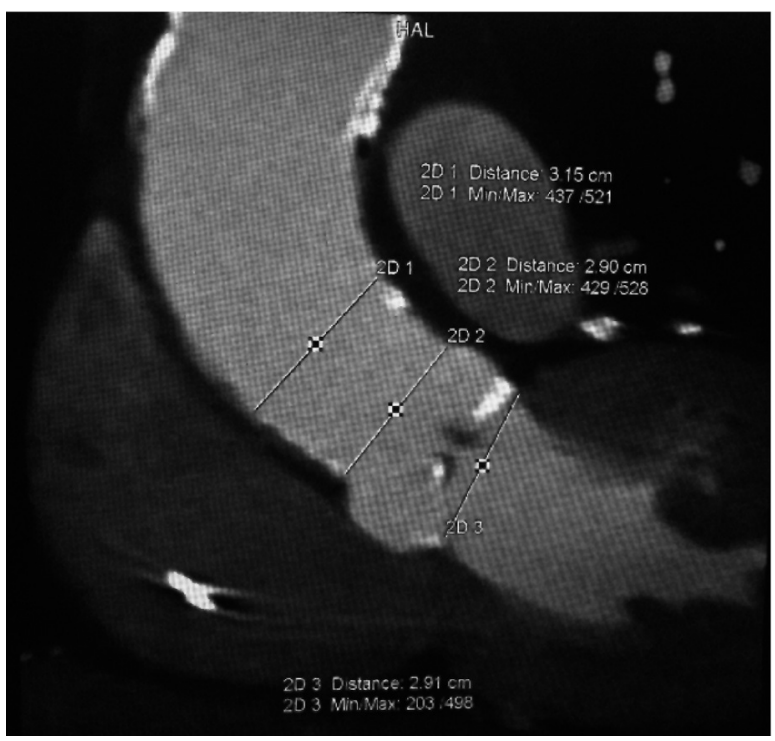

Figura 2. Angiotomografía de aorta torácica: corte a nivel del tracto de salida del ventrículo izquierdo, donde se aprecia las mediciones a nivel del anillo aórtico, unión sinotubular y aorta ascendente, según protocolo requerido. 

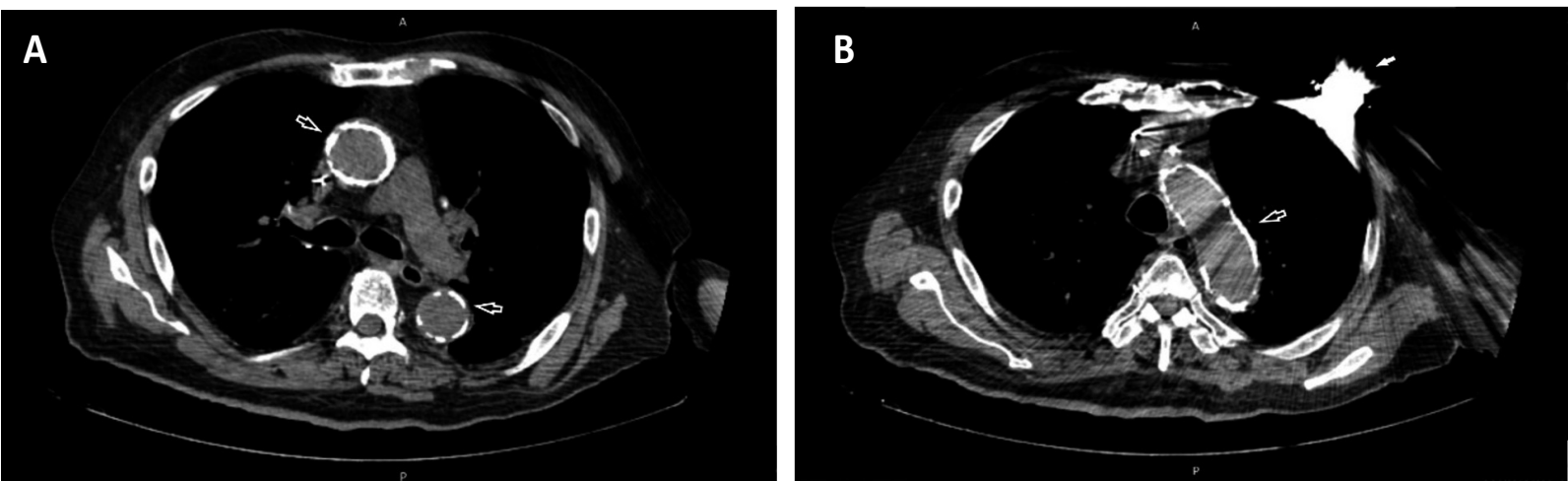

Figura 3. A) Tomografía computarizada de tórax: corte axial a nivel de cayado aórtico, donde se aprecia pared aórtica severamente calcificada (flecha negra). Incidentalmente se aprecia generador de marcapaso definitivo en región pectoral izquierda (flecha blanca). B) Tomografía computarizada de tórax: corte axial a nivel de aorta ascendente y descendente, donde se aprecia paredes aorticas con calcificaciones circunferenciales a predominio de aorta ascendente (flechas negras).

\section{DISCUSIÓN}

El tratamiento recomendado de la estenosis aórtica severa sintomática es el cambio de la válvula afectada, ya sea mediante reemplazo quirúrgico o la colocación de un TAVI. De acuerdo a la guía AHA/ACC ${ }^{[12]}$ de enfermedades cardiacas valvulares nuestro paciente correspondía a una estenosis aórtica severa estadio $D 2$, con un área valvular $<1 \mathrm{~cm}^{2}$, velocidad máxima $<4 \mathrm{~m} / \mathrm{s}$, gradiente máxima $<40 \mathrm{mmHg}$ y $\mathrm{FEVI}<50 \%$; el reemplazo de válvula aórtica tiene una recomendación de clase lla y nivel de evidencia B.

En nuestro paciente no se realizó el estudio de eco estrés con dobutamina, lo que constituyó una limitación del caso. La evaluación del riesgo combinando el puntaje de STS, fragilidad, disfunción sistémica mayor e impedimentos específicos del procedimiento nos arrojó en riesgo prohibitivo de una cirugía de reemplazo de válvula aórtica debido a la calcificación severa de la aorta torácica en toda su extensión.

Se usa el término "aorta en porcelana" cuando existe una extensa calcificación circunferencial o casi circunferencial de la aorta torácica ${ }^{[13,14]}$ y el problema que conlleva es que interfiere con la canulación de la aorta, pinzamiento aórtico, aortotomía o anastomosis de bypass coronario central, requiriendo modificación de la técnica quirúrgica para evitar complicaciones ${ }^{[15]}$. De haber realizado a nuestro paciente una cirugía convencional de reemplazo valvular, el pinzamiento y canulación hubieran causado un daño excesivo a la aorta y/o liberación de material tromboembólico que aumentan el riesgo de que se desarrolle un accidente vascular cerebral.

Por otro lado, nuestro paciente tuvo el diagnóstico de policitemia vera, enfermedad crónica mieloproliferativa caracterizada por una excesiva producción de eritrocitos y, en un $50 \%$ de los casos, de leucocitos y plaquetas ${ }^{[16]}$. Esta enfermedad incrementa el riesgo de eventos trombóticos y hemorrágicos, siendo los predictores mayores de trombosis el tener más de 60 años e historia previa de trombosis, mientras que para eventos hemorrágicos lo es tener un recuento plaquetario mayor de 1500 $\times 10^{9} / L^{[17]}$. Por eso, actualmente se recomienda un hematocrito objetivo $<45 \%$ a fin de evitar las complicaciones relacionadas a la enfermedad ${ }^{[18]}$. Por la edad, nuestro paciente se encontraba dentro del grupo de alto riesgo de complicaciones, además no tenía el valor recomendado de hematocrito.
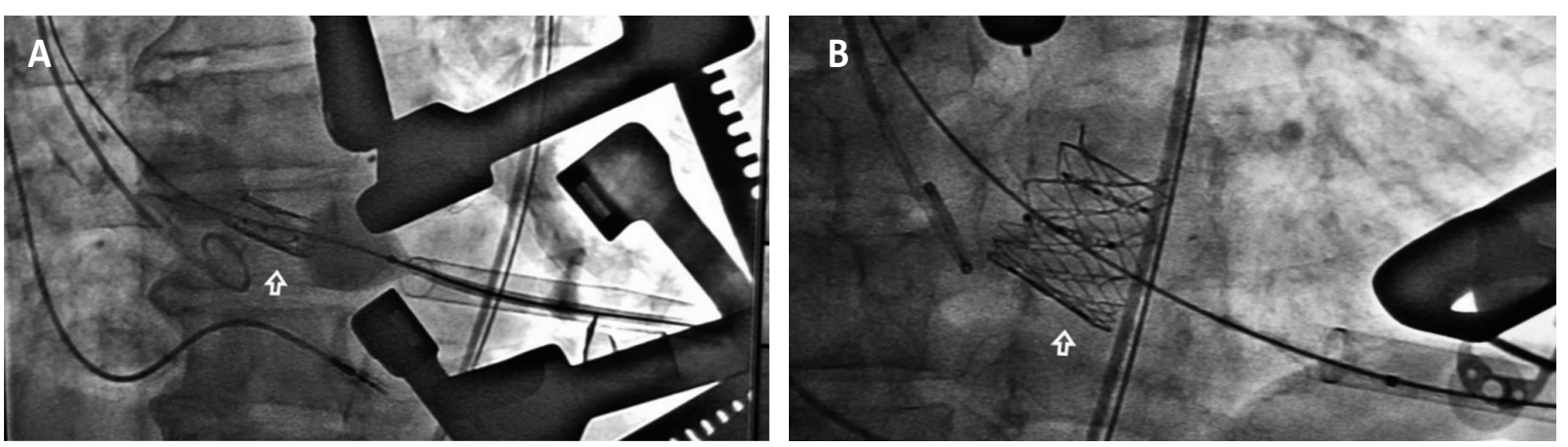

Figura 4. A) Cineangiografía durante liberación de válvula bioprotésica (flecha). B) Cineangiografía de válvula bioprotésica con expansión completa (flecha). 
Se consideró que en nuestro paciente la colocación de TAVI era la mejor opción; se optó por el abordaje transapical debido a que en pacientes con aorta en porcelana se describe el beneficio teórico de este acceso pues se evitaría tocar los vasos femorales, a menudo muy calcificados, y la aorta abdominal, y, lo más importante, habría una menor manipulación del arco aórtico y con ello una menor tasa de accidentes cerebrovasculares ${ }^{[8,19]}$.

Hace poco más de una década los pacientes de alto riesgo o no operables tenían pocas opciones terapéuticas además del cuidado paliativo. Desde entonces se ha visto un aumento exponencial del TAVI, siendo la vía femoral la más usada. Los primeros implantes por vía transapical fueron realizados en el año $2006^{[20]}$ y desde entonces la técnica se ha difundido y estandarizado en el resto del mundo. A nuestro entender, nuestro caso corresponde al noveno procedimiento de TAVI transapical (julio 2017) y es el primero realizado fuera de la ciudad de Lima. La colocación de TAVI constituye una alternativa terapéutica aún novedosa en nuestro medio para aquellos pacientes con estenosis aórtica severa sintomáticos con riesgo alto de mortalidad u otra condición que los hace "inoperables" para una cirugía convencional de reemplazo de válvula aórtica y que tengan alguna limitación para la vía femoral.

Conflicto de interés: Los autores declaran no tener conflicto de interés.

\section{REFERENCIAS BIBLIOGRÁFICAS}

1. Rajamannan NM. Calcific aortic stenosis. Lessons learned from experimental and clinical studies. Arterioscler Thromb Vasc Biol. 2009;29(2):162-8.

2. Goldbarg SH, Elmariah S, Miller MA, Fuster V. Insights into degenerative aortic valve disease. J Am Coll Cardiol. 2007;50(13):1205-13.

3. Nkomo VT, Gardin JM, Skelton TN, Gottdiener JS, Scott CG, EnriquezSarano M. Burden of valvular heart diseases: a population based study. Lancet. 2006;368(9540):1005-11.

4. Lester SJ, Heilbron B, Gin K, Dodek A, Jue J. The natural history and rate of progression of aortic stenosis. Chest. 1998;113(4):1109-14.

5. Schwarz F, Baumann P, Manthey J, Hoffmann M, Schuler G, Mehmel $\mathrm{HC}$, et al. The effect of aortic valve replacement on survival. Circulation. 1982;66(5):1105-10.

6. Otto $\mathrm{CM}$, Kumbhani DJ, Alexander KP, Calhoon JH, Desai MY, Kaul S, et al. 2017 ACC Expert consensus decision pathway for transcatheter aortic valve replacement in the management of adults with aortic stenosis: A report of the american college of cardiology task force on clinical expert consensus documents. J Am Coll Cardiol. 2017;69(10):1313-46.

7. Braxton J, Rasmussen K, Shah M. Transcatheter aortic valve replacement: a review. Surg Clin North Am. 2017;97(4):899-921.

8. Pascual I, Carro A, Avanzas P, Hernández-Vaquero D, Díaz R, Rozado $\mathrm{J}$, et al. Vascular approach for transcatheter aortic valve implantion. J Thorac Dis. 2017;9(Suppl 6):S478-87.

9. Leon MB, Smith CR, Mack MJ, Miller DC, Moses JW, Svensson LG, et al. Transcatheter aortic-valve implantation for aortic stenosis in patients who cannot undergo surgery. N Engl J Med. 2010;363(17):1597-607.

10. Leon MB, Smith CR, Mack MJ, Makkar RR, Svensson LG, Kodali SK, et al. Transcatheter or surgical aortic-valve replacement in intermediate-risk patients. N Engl J Med. 2016;374(17):1609-20.

11. Cribier A, Eltchaninoff $H$, Bash A, Borenstein N, Tron C, Bauer F, et al. Percutaneous transcatheter implantation of an aortic valve prosthesis for calcific aortic stenosis: first human case description. Circulation. 2002;106(24):3006-8.

12. Nishimura RA, Otto CM, Bonow RO, Carabello BA, Erwin JP 3rd, Guyton RA, et al. 2014 AHA/ACC guideline for the management of patients with valvular heart disease a report of the American College of Cardiology/American Heart Association task force on practice guidelines. J Am Coll Cardiol. 2014;63(22):e57-185.

13. Abramovitz $Y$, Jilaihawi $H$, Chacrawarty $T$, Mack MJ, Makkar RR. Porcelain aorta: a comprehensive review. Circulation. 2015;131(9):827-36.

14. Van Mieghem N \& Van Der Boon R. Aorta de porcelana y estenosis aórtica grave: ¿ La implantación percutánea de válvula aórtica es el nuevo tratamiento estándar? Rev Esp Cardiol. 2013;66(10):765-7.

15. Fukuda I, Daitoku K, Minakawa M, Fukuda W. Shaggy and calcified aorta: surgical implications. Gen Thorac Cardiovasc Surg. 2013;61(6):301-13.

16. Landolfi R, Cipriani MC, Novarese L. Thrombosis and bleeding in polycythemia vera and essential thrombocythemia. Pathogenetic machanisms and prevention. Best Pract Res Clin Haematol. 2006;19(3):617-33.

17. Hernandez-Boluda JC, Gómez M. Target hematologic values in the management of essential thrombocythemia and polycythemia vera. Eur J Haematol. 2015;94(1):4-11.

18. Marchioli R, Finazzi G, Specchia G, Cacciola R, Cavazzina R, Cilloni $D$, et al. Cardiovascular events and intensity of treatment in polycythemia vera. N Engl J Med. 2013;368(1):22-33.

19. Kempfert J, Van Linden A, Linke A, Schuler G, Rastan A, Lehmann $S$, et al. Transapical aortic valve implantation: therapy of choice for patients with aortic stenosis and porcelain aorta? Ann Thorac Surg. 2010;90(5):1457-61.

20. Ye J, Cheung A, Lichtenstein SV, Carere RG, Thompson CR, Pasupati $S$, et al. Transapical aortic valve implantation in humans. J Thorac Cardiovasc Surg. 2006;131(5):1194-6. 